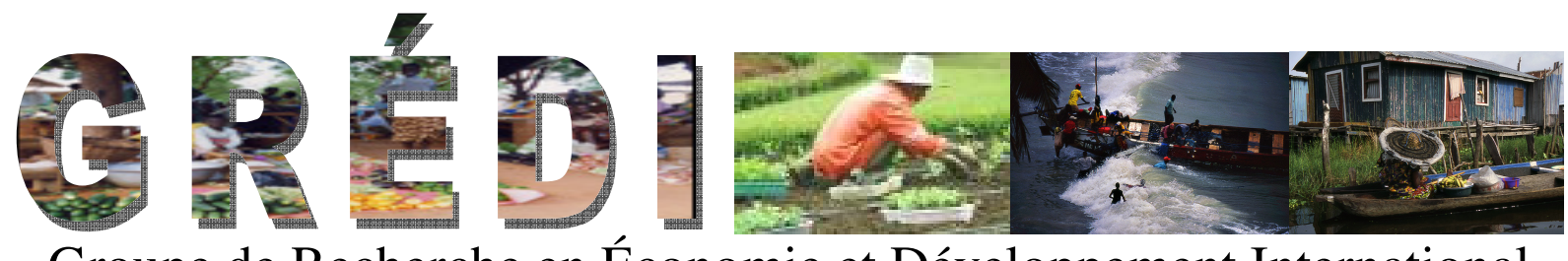

Groupe de Recherche en Économie et Développement International

Cahier de recherche / Working Paper

08-21

Linking Yitzhaki's and Dagum's Gini Decompositions

Stéphane Mussard

Patrick Richard 


\title{
Linking Yitzhaki's and Dagum's Gini Decompositions
}

\author{
Stéphane Mussard* \\ LAmeta-CnRS, Université Montpellier I GRÉDI, Université de Sherbrooke \\ Patrick Richard ${ }^{\dagger}$
}

This version: November 2008

\begin{abstract}
In this article we show that the Gini coefficient is simultaneously decomposable both by sources of income and by populations of income receivers for non-overlapping income distributions: the so-called first-best Gini multi-decomposition. We show that this multidimensional decomposition is useful for many reasons: (i) it is related to the degree of inequality aversion of the decision maker, (ii) it is especially well suited to study inequalities between poor and non-poor people, (iii) it enables one to measure the impact of marginal tax reforms on within- and between-group, respectively.
\end{abstract}

Key words and phrases: Extended Gini, Multidimensional Decomposition, Tax reforms.

JEL Codes: D63, D31.

${ }^{*}$ Lameta, Université Montpellier I, Avenue de la Mer - Site de Richter - C.S. 79606, 34960 Montpellier Cedex 2 France. Tel: 33 (0)467158382 / Fax : 33 (0)4 67158467 - e-mail: smussard@adm.usherbrooke.ca, Associate researcher at GRÉDI, Université de Sherbrooke.

†GRÉDI, Département d'économique, Université de Sherbrooke, 2500, boulevard de l'Université, Sherbrooke, Québec, Canada, J1K 2R1; Email: patrick.richard2@usherbrooke.ca. This research was supported, in part, by a grant from the Fonds Québécois de Recherche sur la Société et la Culture. 


\section{Introduction}

The last few decades have seen the body of literature on inequality measures grow at an impressive rate. Among the numerous innovations to take place in those years, the development of new decomposition techniques has occupied a privileged place, see e.g. Bourguignon (1979), Cowell (1980), Shorrocks (1980, 1982, 1999). Two types of decompositions have been proposed.

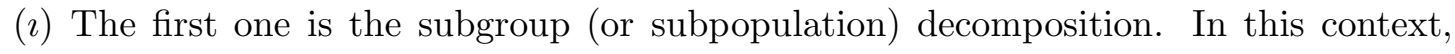
inequality indices yield two components: a within-group term $I_{w}$, that measures inequality within each group of the population, and a between-group term $I_{b}$, which gives an inequality in mean between groups, see e.g. Bourguignon (1979), Shorrocks (1980) and Cowell (1980) for the generalized entropy inequality measure. Other well-known indices such as the Gini index are also decomposable but in a different manner, see e.g. Bhattacharya and Mahalanobis (1967), Rao (1969), Pyatt (1976), Silber (1989), Lerman and Yitzhaki (1991), Lambert and Aronson (1993) and Dagum (1997) among others. Indeed, the structure of the Gini index decomposition is different since it enables one to determine inequality within groups $\left(G_{w}\right)$ and inequality between groups $\left(G_{g b}\right)$, where $G_{g b}$ is an "across group" index of inequality, such that $G=G_{w}+G_{g b} \cdot{ }^{1}$

The index $G_{g b}$ is different from $I_{b}$ in the sense that the former gauges all pairs of income differences between agents' incomes across different groups, whereas the latter gives the inequality between the mean incomes of the groups. Finally, these two measures of inequality between groups provide decision makers a set of two tools to analyze inequalities between subpopulations.

(ı) The second technique of decomposition is the decomposition by income sources (or factor components), which ascribes a part of the overall inequality to each income constituent. The developments about this approach generated a better understanding of the components issued from the Gini index, see e.g. Rao (1969), Fei, Ranis and Kuo (1978), Lerman and Yitzhaki (1985), Shorrocks (1982), and Silber (1993). First, Rao (1969) introduced the concept of pseudo-Gini which he used to decompose the overall Gini index as a weighted average of pseudo-Gini indices by sources of income. This has been the starting point of multiple empirical applications (see e.g. Fei, Ranis and Kuo (1978), Fields (1979)). On the other hand, Shorrocks (1982) generalized the technique of source decomposition

\footnotetext{
${ }^{1}$ When at least two subgroup income distributions overlap, it can be shown that $G=G_{w}+G_{b}+G_{t}$, where $G_{g b}=G_{b}+G_{t}$, and where $G_{b}$ represents the inequality between mean incomes (in the sense of $I_{b}$ ) and $G_{t}$ the inequality of overlap, see e.g. Pyatt (1976), Silber (1989), Lerman and Yitzhaki (1991), Lambert and Aronson (1993) and Dagum (1997) among others. The presence of the ambiguous term $G_{t}$, lead many authors, such as Mookherjee and Shorrocks (1982), to reject the Gini index as a relevant decomposable measure. Since then, researchers have employed the expression "between-group inequality" in order to characterize $I_{b}$ and the expression "across group inequality" (or "gross between-group inequality" in Dagum (1997)) for other related but different measures such as $G_{g b}$. Notice that the $G_{t}$ index is derived from the concept of Gini's (1916) transvariation. The transvariation gauges between-group inequality generated from the groups with lower mean incomes. This means that, when computing inequalities between two or more groups, one assumes that some members of the poorest groups may earn incomes which are greater than those of the members of richest groups.
} 
on the basis of six fundamental axioms in order to derive two fundamental indices: the variance and the coefficient of variation, for which the decomposed elements are identical to those using the Shapley Value on these two indices (Shorrocks, 1999). On the contrary, Lerman and Yitzhaki (1985) pointed out that the extended Gini index provides a desirable decomposition by income sources since the overall extended Gini index provides a wide range of information such as the Gini index for each source of incomes, each source's share in the aggregate income, the correlation between income sources and individuals' ranking within the distribution, and the social preference towards equality, which may be quite important when conducting an empirical investigation since it may help to understand the behavior of the decision maker.

Each type of decompositions motivated its own line of research, but since many years a new field has emerged from these two lines, that of multidimensional decomposition (multi-decomposition for short). This technique aims at combining the two approaches of decomposition in order to jointly identify all inequality components rather than getting them separately through the two decompositions. One of the first attempts is due to Yao (1999) who provides, on the one hand, a subgroup Gini decomposition of the population partitioned into rural and urban areas, and on the other hand, a source decomposition of the within-group inequalities. The breakdown of the Gini coefficient is thus not fully accomplished since the between-group inequality is not decomposed by sources of income. Shorrocks (1999) shows that the Shapley Value enables poverty and inequality measures to be separated both by sources and subgroups. The robustness of this technique relies on its perspective of generalizations. However, the obtained contributions are not necessarily weighted averages of indices defined over the intersecting space of sources and subgroups. The same problem exists with the multi-decomposition of the Gini index and the coefficient of variation squared derived by Mussard (2004, 2006) and Chameni (2007), respectively. One of the aims of this paper is to propose a new Gini multi-decomposition which avoids this problem.

Dagum's and Yitzhaki's contributions to the Gini decompositions are of great interest since they have contributed to remove many doubts about the decomposability of the Gini index (or the extended Gini index in other cases) in its various configurations. Hence, we begin our study by proposing a first-best Gini multi-decomposition based on the technique introduced by these two authors in order to capture new inequality measures. These new measures are related to the aversion of the decision maker towards inequality so that our approach is robust over a large range of decision maker's behavior. Second-best Gini multidecomposition (not treated in this paper) are defined to be a breakdown of the Gini index, which is not exactly a weighted average of Gini indices computed over the space of sources and subgroups simultaneously (see Section 2 infra). In order to obtain a decomposition relevant with the above remarks, and to nest it within the spirit of Lerman and Yitzhaki's (1985) decomposition, the first-best Gini multi-decomposition will be based on the particular but necessary property of non-overlapping income distributions, which helps to focus on the 
problem of how decision makers analyze the income repartition between poor and non poor people. This is another goal of our paper.

To recap, the aim of our paper is four-fold:

- To obtain a multi-decomposition dependent on the social preference towards equality of the social planner (or equivalently her aversion towards inequality).

- To provide a convenient decomposition to carry out robust poor / non-poor analysis given any poverty line. Such partitioning of the population enables one to study a wide range of poverty reducing policies.

- To estimate the contribution of each source, each group and each combination "source/group" to the overall Gini index and calculate their confidence intervals (asymptotic and bootstrap).

- To capture the impacts of tax reforms, for instance subsidizing a particular source of income in any group in order to apprehend the impact on income inequality.

The literature is not silent about the fourth point. Lerman and Yitzhaki (1985) analyse marginal tax reforms through the use of Gini elasticities. These elasticities have been further decomposed by Silber (1998) following the lines of Yitzhaki (2002). ${ }^{2}$ A marginal tax reform gauges the impact of global transfers for a specific source of income (or consumption expenditure) on the overall inequality, that is, the effect of the change in percentage terms in a tax on a particular commodity on the Gini coefficient of income inequality. Yitzhaki's (2002) technique allows the decomposition of the Gini elasticity to be performed in order to apprehend three aspects of elasticities: elasticity among rich, elasticity among poor, elasticity between rich and poor. We follow this idea without exploring elasticities decomposition. We examine marginal tax reforms which are consistent with the extended Gini multi-decomposition implemented in partitioning the population in rich and poor people. As the multi-decomposition yields within-group inequality decomposed by income sources and between-group inequality decomposed by income sources, it provides, for policy purposes, a way to simulate changes in percentage of a tax commodity in one group only (say poor group) in order to capture the effect on within-group inequality or between-group inequality. Hence, the difference with Yitzhaki (2002) relies on the following points.

- The variations of inequalities are computed with the extended Gini index to perform sensitivity analysis. ${ }^{3}$

- The variations of the within-group extended Gini index makes it possible to measure the impact of subsidizing one source in a particular group (admittedly the poor one) on the inequality of this group (and on the overall amount of the within-group inequality).

- Two between-group marginal tax reforms are constructed. The first one yields the impact of subsidizing one income source in the poor group on the inequality between poor and rich (and the impact on the overall between-group inequality). The second one provides a measure of the impact of subsidizing one source in both poor and rich groups on the inequality between rich and poor (and on the overall amount of between-group inequality).

\footnotetext{
${ }^{2}$ Yitzhaki's decomposition was first published as working paper in 1997 and published in 2002.

${ }^{3}$ Yitzhaki (2002, page 71, footnote 19) indicates that a way to increase the weight on poor people is to use the extended Gini index.
} 
The remainder of the paper is organized as follows. Section 2 introduces the concept of multi-decomposition. Section 3 is devoted to the specification of the first-best extended Gini multi-decomposition. Section 4 deals with the derivation of the Gini variations from this first-best multi-decomposition. Section 5 presents an empirical application on Luxembourg data showing, for instance, that subsidizing pension incomes in the poor group has an important effect on the between-group inequality reduction. A brief conclusion follows in Section 6 .

\section{The Gini Multi-decomposition: Notations and Concept}

Let $x_{i k}$ be the income of the $i$ th individual $i \in\{1,2, \ldots, n\}$ belonging to the $k$ th group $\Pi_{k} k \in\{1,2, \ldots, K\}$ of a population divided in $K$ groups. Suppose that total income is disaggregated in sources and let $x_{i k}^{\ell}$ be the income from source $\ell$ of individual $i$, where $\ell \in\{1,2, \ldots, L\} .{ }^{4}$ Let $\mu$ be the average income over the whole population (of size $n$ ) and let $\mu_{k}$ be the average income of individuals in group $\Pi_{k}$ (of size $n_{k}$ ).

The Gini multi-decomposition is a technique by which the subgroup decomposition (Column Total) and the source decomposition (Row Total) are combined to produce a set of new contribution indicators as depicted in Table 1.

Table 1: Structure of the Gini multi-decomposition

\begin{tabular}{|c||c|c|c|c|c||c|}
\hline $\begin{array}{c}\text { Sources } \rightarrow \\
\text { Inequalities } \downarrow\end{array}$ & Source 1 & $\ldots$ & Source $\ell$ & $\ldots$ & Source $L$ & Total \\
\hline \hline Within $\Pi_{1}$ & $C_{11}^{1}$ & $\cdots$ & $C_{11}^{\ell}$ & $\cdots$ & $C_{11}^{L}$ & $C_{11}$ \\
\hline$\vdots$ & $\vdots$ & $\ldots$ & $\vdots$ & $\ldots$ & $\vdots$ & $\vdots$ \\
\hline Within $\Pi_{k}$ & $C_{k k}^{1}$ & $\cdots$ & $C_{k k}^{\ell}$ & $\cdots$ & $C_{k k}^{L}$ & $C_{k k}$ \\
\hline$\vdots$ & $\vdots$ & $\ldots$ & $\vdots$ & $\ldots$ & $\vdots$ & $\vdots$ \\
\hline Within $\Pi_{K}$ & $C_{K K}^{1}$ & $\cdots$ & $C_{K K}^{\ell}$ & $\cdots$ & $C_{K K}^{L}$ & $C_{K K}$ \\
\hline \hline Between $\Pi_{1}$ and $\Pi_{2}$ & $C_{12}^{1}$ & $\cdots$ & $C_{12}^{\ell}$ & $\cdots$ & $C_{12}^{L}$ & $C_{12}$ \\
\hline$\vdots$ & $\vdots$ & $\cdots$ & $\vdots$ & $\cdots$ & $\vdots$ & $\vdots$ \\
\hline Between $\Pi_{k}$ and $\Pi_{j}$ & $C_{k j}^{1}$ & $\cdots$ & $C_{k j}^{\ell}$ & $\cdots$ & $C_{k j}^{L}$ & $C_{k j}$ \\
\hline$\vdots$ & $\vdots$ & $\cdots$ & $\vdots$ & $\cdots$ & $\vdots$ & $\vdots$ \\
\hline Between $\Pi_{K-1}$ and $\Pi_{K}$ & $C_{K-1 K}^{1}$ & $\cdots$ & $C_{K-1 K}^{\ell}$ & $\cdots$ & $C_{K-1 K}^{L}$ & $C_{K-1 K}$ \\
\hline \hline Total & $C^{1}$ & $\cdots$ & $C^{\ell}$ & $\cdots$ & $C^{L}$ & $\sum_{\ell} C^{\ell}=G_{k}$ \\
\hline
\end{tabular}

As shown by Mussard (2006), the within- and across-group contribution indices for income source $\ell$, respectively $C_{k k}^{\ell}$ and $C_{k j}^{\ell}$, are atypical indices since they do not depend on Gini indices for source $\ell$ in each group $\Pi_{k}$ and Gini indices for source $\ell$ across the groups $\Pi_{k}$ and $\Pi_{j}$, namely, $G_{k k}^{\ell}$ and $G_{k j}^{\ell}{ }^{5}$ The contribution indices are based on a different

\footnotetext{
${ }^{4}$ Notice that $x_{i k}$ need not be the income of individual $i$ and could just as well be his total consumption in which case, $x_{i k}^{\ell}$ would be his total consumption of good $\ell$ and all the analysis that follows would bear on consumption rather than income inequality. In this respect, marginal changes in Gini (see Section 4 infra, Lerman and Yitzhaki (1985), Garner (1993)) are also of interest.

${ }^{5}$ These Gini indices are computed on the intersecting space of sources and subgroups. This is not the case when decompositions techniques are separately used.
} 
axiomatic shape, but the proposed decomposition is nevertheless relevant. Indeed, it yields $L\left(K+\frac{K(K-1)}{2}\right)$ combined indices measuring contributions to $G$ which are:

- $C_{k k}^{\ell}$, the contribution of source $\ell$ inequalities (e.g. wages) in group $\Pi_{k}$ to $G$;

- $C_{k j}^{\ell}$, the contribution of source $\ell$ inequalities across groups $\Pi_{k}$ and $\Pi_{j}$ to $G$.

Moreover, the multi-decomposition has the property of independence between the "marginal" decompositions (that is, the decomposition only by income source or by group), meaning that the multi-decomposition can identify $\left(C_{k k}^{\ell}\right)$ as the most contributive index to $G$, whereas the combination of the margins produces another source / group couple. However, the technique may be viewed as a second-best approach since neither the contributions nor the overall Gini index are expressed as a linear combination of within-group Gini coefficients for group $\Pi_{k}$ and source $\ell\left(G_{k k}^{\ell}\right)$ and Gini coefficients across groups $\Pi_{k}$ and $\Pi_{j}$ for source $\ell\left(G_{k j}^{\ell}\right)$. We address this issue in the next section. ${ }^{6}$

\section{On an Extended Gini Multi-decomposition}

Although our multi-decomposition aims at dealing with poor / non poor income repartitions, that is two groups, let us express our result for $K$ non-overlapping income distributions. Let us define the Gini index of the overall population and the Gini index associated with group $\Pi_{k}$ :

$$
G=\frac{\sum_{i=1}^{n} \sum_{r=1}^{n}\left|x_{i}-x_{r}\right|}{2 n^{2} \mu}, G_{k k}=\frac{\sum_{i=1}^{n_{k}} \sum_{r=1}^{n_{k}}\left|x_{i k}-x_{r k}\right|}{2 n_{k}^{2} \mu_{k}} .
$$

Following Dagum (1987), it is possible to measure inequalities between $\Pi_{k}$ and $\Pi_{j}$ using the Gini index across (between) ${ }^{7}$ two populations of income receivers:

$$
G_{k j}=\frac{\sum_{i=1}^{n_{k}} \sum_{r=1}^{n_{j}}\left|x_{i k}-x_{r j}\right|}{n_{k} n_{j}\left(\mu_{k}+\mu_{j}\right)} .
$$

Furthermore, following Dagum (1997), the Gini index is a weighted average of inequalities within groups and inequalities between groups. ${ }^{8}$ After some algebraic manipulations, we obtain:

$$
G=\underbrace{\sum_{k=1}^{K} P_{k}^{2} S_{k k} G_{k k}}_{G_{w}}+\underbrace{2 \sum_{k=2}^{K} \sum_{j=1}^{k-1} P_{j} P_{k} S_{k j} G_{k j}}_{G_{g b}},
$$

where $P_{k}$ is the population share of group $\Pi_{k}\left(P_{k}:=\frac{n_{k}}{n}\right), S_{k k}$ denotes the income share of group $\Pi_{k}\left(S_{k k}:=\frac{\mu_{k}}{\mu}\right)$, and $S_{k j}$ is the joint income share of groups $\Pi_{k}$ and $\Pi_{j}\left(S_{k j}:=\frac{\mu_{k}+\mu_{j}}{\mu+\mu}\right) .{ }^{9}$

\footnotetext{
${ }^{6} \mathrm{It}$ is worth mentioning that $G$ must be linearly connected with $\left(G_{k k}^{\ell}\right)$ and $\left(G_{k j}^{\ell}\right)$ since their axiomatic properties are known and are desirable.

${ }^{7}$ When the Gini index is decomposed by groups, across-group inequality $\left(G_{g b}\right)$ and between-group inequality $\left(G_{b}\right)$ coincides if and only if groups' income distributions do not overlap. This is the case in our multi-decomposition. Therefore, in the sequel, we use the expression between-group inequality.

${ }^{8}$ In Dagum's paper the mean income of the groups are ordered for ease of exposition. Let us recall that this does not constitute a necessary condition to derive the subgroup decomposition.

${ }^{9}$ If the denominator of $S_{k j}$ is $\mu$, one has to delete 2 before the double summation.
} 
This result yields a within-group Gini index $G_{w}$ and a between-group Gini index $G_{g b}$. In what follows, we perform a second level of decomposition along the sources of income. For this purpose, we use the technique introduced by Yitzhaki (1983).

Yitzhaki (1983) introduces the extended Gini index $G(\nu)$, which captures social judgments such as social aversion to inequality:

$$
G(\nu)=1-\nu(\nu-1) \int_{0}^{1}(1-F)^{\nu-2} L(F) d F, \nu>0
$$

where $L(F)$ is the traditional Lorenz curve and $F$ is the cumulative distribution function (c.d.f.) of the population's income. ${ }^{10}$ The higher $\nu$ is, the greater the preference for equality. For all $\nu \in(0,1)$, there is preference for inequality. If $\nu=1$, there is indifference for inequality $(G(1)$ is always 0$)$. If $\nu=2$ we get the standard Gini index (1) and if $\nu>2$, there is stronger preference for equality. Let $G^{\ell}(\nu)$ be the extended Gini index computed on $x^{\ell}$, the vector of income from the $\ell$ th source:

$$
G^{\ell}(\nu)=\frac{-\nu \operatorname{Cov}\left(x^{\ell},\left(1-F^{\ell}\right)^{\nu-1}\right)}{\mu^{\ell}},
$$

where $\mu^{\ell}$ and $F^{\ell}$ are respectively the mean and c.d.f. of income source $\ell$. Let $R^{\ell}(\nu)$ be the Gini-correlation coefficient measuring the correlation between the $\ell$ th source and aggregate income:

$$
R^{\ell}(\nu)=\frac{\operatorname{Cov}\left(x^{\ell},(1-F)^{\nu-1}\right)}{\operatorname{Cov}\left(x^{\ell},\left(1-F^{\ell}\right)^{\nu-1}\right)}
$$

$R^{\ell}(\nu)$ is a Pearson-like coefficient since its denominator is the covariance between the vector of the $\ell$ th source and the ranks of its elements in the distribution instead of a product of standard deviations. Let $S^{\ell}:=\frac{\mu^{\ell}}{\mu}$ be the income share of source $\ell$. Then, the extended Gini index is source decomposable :

$$
G(\nu)=\sum_{\ell=1}^{L} R^{\ell}(\nu) G^{\ell}(\nu) S^{\ell} .
$$

In order to obtain the multi-decomposition, let us introduce the Gini-correlation index of source $\ell$ for group $k$ and the extended Gini index of source $\ell$ for group $k$ :

$$
R_{k k}^{\ell}(\nu)=\frac{\operatorname{Cov}\left(x_{k}^{\ell},\left(1-F_{k}\right)^{\nu-1}\right)}{\operatorname{Cov}\left(x_{k}^{\ell},\left(1-F_{k}^{\ell}\right)^{\nu-1}\right)}, G_{k k}^{\ell}(\nu)=\frac{-\nu \operatorname{Cov}\left(x_{k}^{\ell},\left(1-F_{k}^{\ell}\right)^{\nu-1}\right)}{\mu_{k}^{\ell}},
$$

where $x_{k}^{\ell}$ is the vector of source $\ell$ for group $k, F_{k}^{\ell}$ is the c.d.f. of source $\ell$ for group $k$ and $\mu_{k}^{\ell}$ is the mean of source $\ell$ for group $k$. Let $S_{k k}^{\ell}:=\frac{\mu_{k}^{\ell}}{\mu_{k}}$ be the $\ell$ th income source share of

\footnotetext{
${ }^{10}$ As shown by Fei, Ranis, and Kuo (1978), F can be replaced by the individuals' position when they are ranked by ascending order of incomes.
} 
group $k$. Then, $G_{k k}(\nu)$ possesses the same structure as $G(\nu)$ :

$$
G_{k k}(\nu)=\sum_{\ell=1}^{L} R_{k k}^{\ell}(\nu) G_{k k}^{\ell}(\nu) S_{k k}^{\ell}
$$

Now, suppose we have $K$ non-overlapping subgroup income distributions. The Gini index (2) between groups $\Pi_{k}$ and $\Pi_{j}$ can be rewritten as:

$$
G_{k j}=\frac{\left|\mu_{k}-\mu_{j}\right|}{\mu_{k}+\mu_{j}} .
$$

Further, the decomposition of $G_{k j}$ by income sources is:

$$
G_{k j}=\sum_{\ell=1}^{L} S_{k j}^{\ell} G_{k j}^{\ell}
$$

where $G_{k j}^{\ell}$ is the Gini index between groups $\Pi_{k}$ and $\Pi_{j}$ for source $\ell$ and $S_{k j}^{\ell}$ is the contribution of source $\ell$ in the means of $\Pi_{k}$ and $\Pi_{j}$ :

$$
G_{k j}^{\ell}=\frac{\left|\mu_{k}^{\ell}-\mu_{j}^{\ell}\right|}{\mu_{k}^{\ell}+\mu_{j}^{\ell}}, S_{k j}^{\ell}=\frac{\mu_{j}^{\ell}+\mu_{k}^{\ell}}{\mu_{j}+\mu_{k}} .
$$

It is worth mentioning that (11) is valid if and only if $\operatorname{sgn}\left(\mu_{k}^{\ell}-\mu_{j}^{\ell}\right)=\operatorname{sgn}\left(\mu_{k}-\mu_{j}\right)$ for all $\ell \in\{1,2, \ldots, L\} .{ }^{11}$ Substituting (9) and (11) in (3) and letting $\nu=2$, one finds that the standard Gini index is multi-decomposable in the sense that it is simultaneously decomposable by income sources and groups of income receivers:

$$
G(2)=\sum_{k=1}^{K} \sum_{\ell=1}^{L} P_{k}^{2} S_{k k} R_{k k}^{\ell}(2) G_{k k}^{\ell}(2) S_{k k}^{\ell}+\sum_{k=2}^{K} \sum_{j=1}^{k-1} \sum_{\ell=1}^{L} 2 P_{j} P_{k} S_{k j} S_{k j}^{\ell} G_{k j}^{\ell} .
$$

As can be seen in (4), the degree of inequality aversion only enters through its interaction with the c.d.f. of incomes. In particular, $\nu$ only affects $G(\nu)$ through the weight that is given to each Lorenz curve ordinate. Specifically, it does not affect the value of these ordinates, which represent the mean income share held by a percentage of the population. On the other hand, non-overlapping subgroup distributions entail between-group inequalities depending only on mean income shares. Thus, between-group inequalities do not depend on the degree of inequality aversion. Hence, the Gini multi-decomposition (13) can naturally be extended for all $\nu>0$ as:

$$
G(\nu)=\underbrace{\sum_{k=1}^{K} \sum_{\ell=1}^{L} P_{k}^{2} S_{k k} R_{k k}^{\ell}(\nu) G_{k k}^{\ell}(\nu) S_{k k}^{\ell}}_{G_{w}(\nu)}+\underbrace{\sum_{k=2}^{K} \sum_{j=1}^{k-1} \sum_{\ell=1}^{L} 2 P_{j} P_{k} S_{k j} S_{k j}^{\ell} G_{k j}^{\ell}}_{G_{g b}} .
$$

\footnotetext{
${ }^{11}$ In a poor / non-poor partition, the condition is usually easily satisfied, meaning that all mean income sources are likely to be greater in the non-poor group than in the poor one.
} 
The extended Gini $(G(\nu))$, the extended within-group Gini $\left(G_{w}(\nu)\right)$, and the between-group Gini $\left(G_{g b}\right)$ can easily be broken down so that Table 1 is fully complete. The extended Gini multi-decomposition then yields the contribution of inequality in group $\Pi_{k}$ due to source $\ell \forall k \in\{1,2, \ldots, K\}$ and $\forall \ell \in\{1,2, \ldots, L\}$ and the contribution of inequality between groups $\Pi_{k}$ and $\Pi_{j} \forall k \neq j \in\{1,2, \ldots, K\}$ due to source $\ell \forall \ell \in\{1,2, \ldots, L\}$, respectively:

$$
C_{k k}^{\ell}(\nu)=P_{k}^{2} S_{k k} R_{k k}^{\ell}(\nu) G_{k k}^{\ell}(\nu) S_{k k}^{\ell}, C_{j k}^{\ell}=2 P_{j} P_{k} S_{k j} S_{j k}^{\ell} G_{k j}^{\ell} \text {. }
$$

At this stage, one remarks that only within-group contributions $\left(C_{k k}^{\ell}(\nu), \forall \nu>0, \forall \ell\right)$ depend on the aversion degree towards inequality. Accordingly, overall inequality may be seen as an increasing function of the deprivation feeling within each group. ${ }^{12}$ Indeed, deprivation is closely related to social exclusion with respect to the reference group, namely, the perception of group exclusion (see Bossert and D'Ambrosio (2006)). In a poor/non-poor framework (see Section 4 infra), poor persons feel deprived or excluded only through their evaluation of their own position in the group they belong to. This situation is also relevant with the literature on poverty measurement, in which the level of poverty does not depend on incomes above a predefined poverty line (the Focus axiom). Therefore, poor individuals are indifferent to what happens in the rich group, and vice versa, so that a higher $\nu$ affects $G(\nu)$ through $G_{w}(\nu)$ only.

\section{Multi-decomposition and Tax Reforms}

The impact of tax reforms on poverty or inequality indices yields decision makers the ability to simulate transfers (see e.g. Duclos, Makdissi and Wodon (2005) for the case of direct transfer reforms, Makdissi and Wodon (2007) to regulatory reforms). In this Section, we study the effect of the change in the tax on a given commodity on the extended Gini coefficient (see also the technique initiated by Lerman and Yitzhaki (1985)). Traditionally, in the literature of inequality measurement, transfers are concerned with one income donor and one income recipient in order to analyze the impact of such transfers on the overall inequality. In contrast, the effect of marginal tax variations on commodities are equivalent to global transfers in income source distributions. For instance, when the population is partitioned in poor and non-poor groups, decision makers may be interested in capturing the impact of a percentage change in each individual's income source $\ell$ on the inequality in each group. ${ }^{13}$ These tax reforms can readily be computed in the case of the extended Gini multi-decomposition. Suppose each individual's source $\ell$ in group $\Pi_{k}$ is multiplied by $e_{k k}^{\ell} \rightarrow 1^{+}$. The extended Gini variation within group $\Pi_{k}$ with respect to source $\ell$ is (see the

\footnotetext{
${ }^{12}$ See Yitzhaki (1979) for the intimate interrelation between the Gini index and concept of relative deprivation.

${ }^{13}$ In what follows, we restrict our attention to marginal variations in the income source distributions (which may be interpreted as marginal taxation). Indeed, marginal tax reforms are supposed not to alter the composition of the poor and non-poor groups. This assumption allows us to avoid the use of dynamic (and over complicated) models, which analyze taxation and the distribution of poor and non-poor people over time.
} 
Appendix):

$$
\mathcal{G}_{k k}^{\ell}(\nu):=\frac{\partial G_{k k}(\nu)}{\partial e_{k k}^{\ell}} \cong S_{k k}^{\ell}\left(R_{k k}^{\ell}(\nu) G_{k k}^{\ell}(\nu)-G_{k k}(\nu)\right)
$$

Notice that these marginal variations may be connected with within-group Gini elasticities:

$$
\frac{\mathcal{G}_{k k}^{\ell}(\nu)}{G_{k k}(\nu)} \cong S_{k k}^{\ell}\left(\eta_{k k}(\nu)-1\right),
$$

where

$$
\eta_{k k}(\nu)=\frac{R_{k k}^{\ell}(\nu) G_{k k}^{\ell}(\nu)}{G_{k k}(\nu)}
$$

is the extended Gini elasticity within group $\Pi_{k}$ (see also Lerman and Yitzhaki, 1985, for the definition). These marginal changes $\left(\mathcal{G}_{k k}^{\ell}(\nu), \forall \nu>0, \forall \ell \in\{1,2, \ldots, L\}\right)$ are useful indicators for the analysis of the impact of tax reforms intending to improve transfers in particular groups or, if $x_{i k}$ represents individual $i$ 's consumption, to subsidize some commodities in order to reduce inequalities among poor people.

As the multi-decomposition methodology is well suited to analyze the behavior of individuals when they differ in needs by means of between-group indicators, it may be of interest to extend the notion of marginal changes when two groups are subsidized simultaneously. For this purpose, it is possible to compute a between-group indicator to capture the effect of the tax reform by assuming that source $\ell$ of each person belonging to group $\Pi_{k}$ and $\Pi_{j}$ is multiplied by $e_{k j}^{\ell} \rightarrow 1^{+}$(see the Appendix):

$$
\mathcal{G}_{k j}^{\ell}:=\frac{\partial G_{k j}}{\partial e_{k j}^{\ell}} \cong S_{k j}^{\ell}\left(G_{k j}^{\ell}-G_{k j}\right) .
$$

Such taxation schemes may be implemented to alleviate inequalities between poor and nonpoor groups. Alternatively, the technique may be employed to subsidize poor and extremely poor groups in order to capture the effect on the inequalities between poor and extremely poor persons.

In a similar manner, it may be interesting to measure the decline of inequalities between $\Pi_{k}$ and $\Pi_{j}$ when poor people alone are receiving with such transfers. Let $K=2$ and assume $\Pi_{k}$ represents the group of poor individuals and suppose that each poor person's source $\ell$ is multiplied by $e_{k k}^{\ell} \rightarrow 1^{+}$(see the Appendix):

$$
\widetilde{\mathcal{G}}_{k j}^{\ell}:=\frac{\partial G_{k j}}{\partial e_{k k}^{\ell}} \cong-\frac{\mu_{k}^{\ell}}{\mu_{j}+\mu_{k}}\left(1+G_{k j}\right) .
$$

Equation (20) implies that subsidizing source $\ell$ in the poor group always decreases inequalities between poor and non poor. ${ }^{14}$

\footnotetext{
${ }^{14}$ Notice that all variations can be measured in proportion of $G_{k k}(\nu)$ or $G_{k j}$ in order to compute percentages.
} 
The above Gini variations allow to capture the impact of global transfers either on the inequalities in one group or on the inequalities between two non-overlapping groups. We now examine the possibility to subsidize the poor and to gauge inequality variations relevant with the extended Gini multi-decomposition. Suppose that the decision maker intends to subsidize the poor group $\Pi_{k}$ by increasing each poor person's source $\ell$. Then, measuring the impact of this policy on $G(\nu)$ necessitates that we evaluate the variation of the contributions $C_{k k}^{\ell}(\nu)$ and $C_{j k}^{\ell}$ issued from the multi-decomposition. ${ }^{15}$ Suppose source $\ell$ is multiplied by $e_{k k}^{\ell} \rightarrow 1^{+}$. Then:

$$
\mathcal{G}^{\ell}:=\frac{\partial G(\nu)}{\partial e_{k k}^{\ell}}=\underbrace{\frac{\partial C_{k k}^{\ell}}{\partial e_{k k}^{\ell}}+\sum_{h \neq \ell} \frac{\partial C_{k k}^{h}}{\partial e_{k k}^{\ell}}+\frac{\partial C_{j j}^{\ell}}{\partial e_{k k}^{\ell}}+\sum_{h \neq \ell} \frac{\partial C_{j j}^{h}}{\partial e_{k k}^{\ell}}}_{\frac{\partial G_{w}(\nu)}{\partial e_{k k}^{\ell}}}+\underbrace{\frac{\partial C_{j k}^{\ell}}{\partial e_{k k}^{\ell}}+\sum_{h \neq \ell} \frac{\partial C_{j k}^{h}}{\partial e_{k k}^{\ell}}}_{\frac{\partial G_{g b}}{\partial e_{k k}^{\ell}}} .
$$

Thus, the impact of the change of source $\ell$ in group $\Pi_{k}$ on the overall inequality $G(\nu)$ is decomposed in two marginal changes. The first one, $\mathcal{G}_{w}^{\ell}$, measures the impact of the variation of source $\ell$ in group $\Pi_{k}$ on the within-group inequality (see the Appendix):

$$
\begin{aligned}
\mathcal{G}_{w}^{\ell}:=\frac{\partial G_{w}(\nu)}{\partial e_{k k}^{\ell}}= & P_{k}^{2} \frac{\mu_{k}^{\ell}}{\mu}\left(1-P_{k} S_{k k}\right) G_{k k}(\nu)+P_{k}^{2} S_{k k} S_{k k}^{\ell}\left(R_{k k}^{\ell}(\nu) G_{k k}^{\ell}(\nu)-G_{k k}(\nu)\right) \\
& -P_{j}^{2} P_{k} S_{j j} \frac{\mu_{k}^{\ell}}{\mu} G_{j j}(\nu) .
\end{aligned}
$$

The second one, $\mathcal{G}_{g b}^{\ell}$, measures the impact of the change of source $\ell$ in group $\Pi_{k}$ on the between-group inequality (see the Appendix):

$$
\mathcal{G}_{g b}^{\ell}:=\frac{\partial G_{g b}}{\partial e_{k k}^{\ell}}=-2 P_{j} P_{k} \frac{\mu_{k}^{\ell}}{2 \mu}\left(1+2 P_{k} S_{j k} G_{j k}\right) .
$$

Finally, decision makers have the ability to subsidize poor groups to reduce poverty, but this kind of policies have many implications on inequalities particularly on within- and between-group inequality components as shown by equations (22) and (23).

\section{Empirical Illustration}

We illustrate the utility of the multi-decomposition using Luxembourg survey data from the Panel Socio-Economique Liewen zu Letzebuerg II (PSELL II) 2004. Our data set is composed of 2,295 households reporting positive incomes in at least one of 5 categories which are defined in Table 2. We consider two groups of households: poor and non-poor ones, where a household is considered poor if its total income is below 0.6 times the sample median total income. This yields a poor group of 398 households and a non-poor group of 1,897 households.

\footnotetext{
${ }^{15}$ This case was studied by Yitzhaki (2002) except we use a multidimensional extended Gini multidecomposition, and except we subsidize source $\ell$ for the poor group only, instead of subsidizing source $\ell$ for the entire population.
} 
Table 2: Income sources

\begin{tabular}{l|l}
\hline Source 1 & Wages and commercial incomes \\
\hline Source 2 & Total transfers income \\
\hline Source 3 & Unemployment insurance income \\
\hline Source 4 & Pension income \\
\hline Source 5 & Other incomes \\
\hline
\end{tabular}

Table 3 shows the estimated contributions to the Gini index when $\nu=2(G(2)=0.3094)$ along with standard normal and bootstrap 95\% confidence intervals. The decomposition by income sources indicates that almost all of the inequality comes from source 1 (0.2503). This is not surprising because this source is composed of all labour incomes, which account for a large proportion of the total income of several households. The multi-decomposition allows us to see that this source's contribution largely comes from within the non-poor group (0.1620), although the between-group contribution is also quite large (0.0879).

The decomposition by groups reveals that practically all the inequality comes from within the non-poor group (0.2007) and between the two groups (0.1072). Thus, it would appear that there is very little inequality in the poor segment of the population, a lot in the non-poor segment, which is mostly due to income source 1 but also to sources 2 and 5 , and also a lot between the poor and non-poor groups, due to the same sources.

It may at first appear odd that some within-group contributions take negative values. This happens whenever $R^{\ell}(2)$ is negative, which implies that $\operatorname{Cov}\left(x_{k}^{\ell},\left(1-F_{k}\right)\right)$ is positive. It therefore makes sense for the contributions of pension and unemployment incomes in the non-poor group to be negative. Indeed, these are on average smaller than employment income, which constitutes the larger share of total incomes, and people who receive them usually are not working. Thus, individuals with positive pension or unemployment income $\left(x^{\ell}>0\right)$ are likely to be ranked in the lower part of the overall distribution (that is, they have a $1-F$ close to 1$)$. On the other hand, people who work typically have no pension or unemployment insurance income $\left(x^{\ell}=0\right)$ but their total income tends to be larger (they have low $1-F)$. Hence, the covariance between $(1-F)$ and $x^{\ell}$ is positive. The same argument can be made for all $\nu \geq 1$ and a similar argument holds for all $0<\nu<1$ (that is, $C_{k k}^{\ell}(0.5)$ is positive whenever $R^{\ell}(0.5)$ is negative because $G_{k k}^{\ell}(0.5)$ is always negative). It also makes a lot of intuitive sense. Indeed, when one income source representing a large share of total income (here, source 1) is distributed with a certain degree of inequality, while another less important source tends to contribute a larger share to the total income of people with low income from that source, then this second income source tends to decrease total inequality, which translates in a negative $C_{k k}^{\ell}$. There is a number of situations in which between-group contributions may also be negative. For example, when $K=2$, if $\mu_{j}^{\ell}<0$, where $j$ denotes the poor group, then $C_{j k}^{\ell}<0 .{ }^{16}$

\footnotetext{
${ }^{16}$ Notice that the between-group contributions $C_{j k}^{\ell}$ cannot be negative if and only if there are only two groups of income earners. If $K>2$, then between-group contributions may be negative.
} 
Table 3. Gini Contributions to $G(\nu)(\nu=2)$

\begin{tabular}{l|c|c|c|c}
\hline & Poor & Non-poor & Between & Sum \\
\hline Source 1 & 0.0004 & 0.1620 & 0.0879 & $\mathbf{0 . 2 5 0 3}$ \\
& $(0.0000,0.0007)$ & $(0.1519,0.1722)$ & $(0.0839,0.0919)$ & \\
& {$[0.0000,0.0007]$} & {$[0.1519,0.1723]$} & {$[0.0839,0.0919]$} & \\
\hline Source 2 & 0.0002 & 0.0282 & 0.0114 & $\mathbf{0 . 0 3 9 8}$ \\
& $(0.0000,0.0004)$ & $(0.0166,0.0398)$ & $(0.0084,0.0143)$ & \\
& {$[0.0000,0.0004]$} & {$[0.0154,0.0385]$} & {$[0.0082,0.0143]$} & \\
\hline Source 3 & -0.0001 & -0.0005 & 0.0008 & $\mathbf{0 . 0 0 0 2}$ \\
& $(-0.0002,-0.0001)$ & $(-0.0011,0.0001)$ & $(0.0001,0.0015)$ & \\
& {$[-0.0002,-0.0001]$} & {$[-0.0011,0.0001]$} & {$[0.0000,0.0014]$} & \\
\hline Source 4 & 0.0010 & -0.0089 & 0.0004 & $\mathbf{- 0 . 0 0 7 5}$ \\
& $(0.0006,0.0013)$ & $(-0.0144,-0.0034)$ & $(-0.0018,0.0025)$ & \\
& {$[0.0006,0.0013]$} & {$[-0.0144,-0.0035]$} & {$[-0.0033,0.0007]$} & \\
\hline Source 5 & 0.0000 & 0.0199 & 0.0067 & $\mathbf{0 . 0 2 6 6}$ \\
& $(0.0000,0.0001)$ & $(0.0133,0.0265)$ & $(0.0052,0.0082)$ & \\
& {$[0.0000,0.0001]$} & {$[0.0127,0.0261]$} & {$[0.0051,0.0081]$} & \\
\hline Sum & $\mathbf{0 . 0 0 1 5}$ & $\mathbf{0 . 2 0 0 7}$ & $\mathbf{0 . 1 0 7 2}$ & $\mathbf{0 . 3 0 9 4}=G(2)$ \\
\hline \multicolumn{7}{|c|}{ Normal confidence intervals in parenthesis } & \\
& Bootstrap confidence intervals in brackets, B=12 000 & \\
&
\end{tabular}

It is interesting to notice that the bootstrap and standard normal confidence intervals are almost identical for all contributions. This is an important result because it tells us something about the asymptotic characteristics of $C_{k k}^{\ell}$ and $C_{j k}^{\ell}$. Indeed, although asymptotic normality results are available for the standard Gini index $G(2)$ (see, among others, Davidson (2007) and Cowell (1989)) or the extended Gini G( $\nu$ ) (see Barrett and Pendakur (1995)) no such results exist for the decomposition (3) nor, obviously, for the multi-decomposition (14). Although no general conclusion may be drawn from them, the results in Table 3 (and Table 4 ) indicate that the $C_{j k}^{\ell}$ 's (for all $j=k$ or $j \neq k$ ) may have asymptotically normal distributions.

The second and third columns of Table 4 show that a higher level of inequality aversion yields very similar results. The magnitude of all the contributions increase, but by different factors. Since the Gini index with $\nu=4$ puts more weight on low incomes (relatively to $\nu=2$ ), this reflects the differences in the depth of inequality (in the sense of the distance between the income of the poorest members of a group and the income of other members of this group) in each group for each income source. For example, the depth of inequality seems to be greater for source 4 than for source 5 in the non-poor group, because the contribution of source 4 increases by a much greater factor than that of source 5 . 
Table 4. Within-group contributions $(\nu=4$ and $\nu=0.5)$

\begin{tabular}{l|c|c|c|c}
\hline & Poor $(\nu=4)$ & Non-poor $(\nu=4)$ & Poor $(\nu=0.5)$ & Non-poor $(\nu=0.5)$ \\
\hline Source 1 & 0.0009 & 0.2607 & -0.0002 & -0.2058 \\
& $(0.0003,0.0014)$ & $(0.2464,0.2750)$ & $(-0.0007,0.0003)$ & $(-0.2548,-0.1569)$ \\
& {$[0.0003,0.0014]$} & {$[0.2464,0.2748]$} & {$[-0.0006,0.0004]$} & {$[-0.2518,-0.1534]$} \\
\hline Source 2 & 0.0004 & 0.0376 & 0.0000 & -0.2261 \\
& $(0.0000,0.0007)$ & $(0.0253,0.0498)$ & $(-0.0002,0.0002)$ & $(-0.3525,-0.0997)$ \\
& {$[0.0000,0.0007]$} & {$[0.0243,0.0488]$} & {$[-0.0002,0.0002]$} & {$[-0.4093,-0.1805]$} \\
\hline Source 3 & -0.0004 & -0.0008 & 0.0000 & 0.0008 \\
& $(-0.0006,-0.0001)$ & $(-0.0020,0.0005)$ & $(0.0000,0.0002)$ & $(0.0004,0.0012)$ \\
& {$[-0.0005,-0.0001]$} & {$[-0.0020,0.0005]$} & {$[0.0000,0.0002]$} & {$[0.0004,0.0012]$} \\
\hline Source 4 & 0.0018 & -0.0202 & -0.0010 & 0.0021 \\
& $(0.0013,0.0024)$ & $(-0.0303,-0.0101)$ & $(-0.0016,-0.0005)$ & $(-0.0089,0.0130)$ \\
& {$[0.0013,0.0024]$} & {$[-0.0302,-0.0102]$} & {$[-0.0018,-0.0007]$} & {$[-0.0074,0.0148]$} \\
\hline Source 5 & 0.0000 & 0.0247 & 0.0000 & -0.0785 \\
& $(0.0000,0.0001)$ & $(0.0176,0.0318)$ & $(-0.0001,0.0000)$ & $(-0.1273,-0.0296)$ \\
& {$[0.0000,0.0001]$} & {$[0.0171,0.0312]$} & {$[-0.0001,0.0000]$} & {$[-0.1235,-0.0265]$} \\
\hline \multicolumn{5}{c}{ Normal confidence intervals in parenthesis } \\
\hline
\end{tabular}

Things are quite different when there is preference for inequality (columns 4 and 5). The first change is that all the statistically significant contributions have an opposite sign compared with those computed with inequality aversion $(\nu=2$ and $\nu=4)$. This is not a surprise and confirms that the multi-decomposition works well. The contribution of source 2 in the non-poor group, which was modest with inequality aversion, is now very large and comparable to that or source 1. Further, this is the only case where the bootstrap confidence interval substantially differs from the normal one. It is possible that this results from the fact that $\nu=0.5$ puts more weight on large incomes and may have amplified a heavy tail effect for this source. This conjecture is based on the fact that income source 2 in the non-poor group has a much higher kurtosis $(\kappa=439)$ than any other income sourcegroup combination ( $\kappa=198$ for non-poor source 5 is the second highest). It is well known that the standard bootstrap can be quite inaccurate, and even invalid, when it is used with data from a distribution with heavy tails (of course, standard normal approximations are also quite inaccurate in such cases). Bootstrap methods designed to yield valid and more reliable finite sample inferences for inequality measures in data sets with heavy tails exist, see Davidson and Flachaire (2007). Adapting those to the multi-decomposition is beyond the scope of this article and is left to future work.

The within- and between-group marginal changes (equations 16, 19 and 20) are displayed in Table 5. Several interesting results are worth highlighting. In particular, the table suggests that subsidizing income sources 1 and 4 in the poor group has an important inequality reducing effect through the between-group marginal variation $\tilde{\mathcal{G}}_{k j}^{\ell}$. Also, an inequality averse government could significantly reduce within-group inequality by, for example, subsidizing members of the poor group through an increase of their incomes from source 1 or taxing income source 1 of members of the non-poor group. In addition, a substantial between-group inequality reduction is achieved by increasing source 4 incomes in both groups simultaneously. 
Some further insight about the Gini variations may be gained from a close examination of the results in Table 5. For instance, notice that, for wages and commercial incomes (source 1), $\mathcal{G}_{k j}^{\ell}>0$ while $\widetilde{\mathcal{G}}_{k j}^{\ell}<0$. This mainly reflects the fact that $57 \%$ of the members of the poor group have no source 1 income while only $18 \%$ of the members of the non-poor group are in that situation. Thus, a policy which increases income source 1 for all members of the population has a much larger impact on the mean income of the non-poor group than on that of the poor group, and consequently has a positive net effect on between-group inequality. Hence, $\mathcal{G}_{k j}^{\ell}$ is positive. On the other hand, increasing source 1 incomes only for members of the poor group obviously reduces between-group inequality, hence $\widetilde{\mathcal{G}}_{k j}^{\ell}$ is negative.

Table 5. Within- and between-group inequality variations by groups and income sources

\begin{tabular}{l|c|c|c|c|c|c|c|c}
\hline & $\mathcal{G}_{p}^{\ell}(0.5)$ & $\mathcal{G}_{n p}^{\ell}(0.5)$ & $\mathcal{G}_{p}^{\ell}(2)$ & $\mathcal{G}_{n p}^{\ell}(2)$ & $\mathcal{G}_{p}^{\ell}(4)$ & $\mathcal{G}_{n p}^{\ell}(4)$ & $\mathcal{G}_{k j}^{\ell}$ & $\tilde{\mathcal{G}}_{k j}^{\ell}$ \\
\hline Source 1 & 0.0213 & 0.1735 & -0.0134 & 0.0361 & -0.0119 & 0.0762 & 0.1181 & -0.1379 \\
Source 2 & 0.0122 & -0.2171 & 0.0019 & 0.0064 & 0.0000 & 0.0033 & -0.0060 & -0.0504 \\
Source 3 & 0.0100 & 0.0040 & -0.0159 & -0.0018 & -0.0362 & -0.0028 & -0.0014 & -0.0102 \\
Source 4 & -0.0405 & 0.1105 & 0.0251 & -0.0542 & 0.0449 & -0.0904 & -0.1152 & -0.1778 \\
Source 5 & -0.0029 & -0.0709 & 0.0022 & 0.0136 & 0.0032 & 0.0137 & 0.0117 & -0.0063 \\
\hline
\end{tabular}

Table 6 provides Gini variations measuring the impact of source changes for the poor group on overall inequality (21), on within-group inequalities (22), and on between-group inequalities (23). According to the numbers depicted there, increasing poor group's pensions (source 4) yields the most important reduction of between-group inequalities, of withingroup inequalities (for $\nu=2$ and $\nu=4$ ), and of the overall inequality. Pensions are also the most contributive source that raises within-group inequalities when there is preference for inequality $(\nu=0.5)$.

Finally, increasing any one of the poor group's income source decreases inequality no matter what the degree of inequality aversion is. The main driving force of this is the between-group effect. In particular, the between-group variations are strong enough to offset the positive within-group variation $\mathcal{G}_{w}^{\ell}(0.5)$, so that the impact on the overall inequality is always negative.

Table 6. Within- and between-group inequality variations by income sources

\begin{tabular}{l|c|c|c|c|c|c|c}
\hline & $\mathcal{G}_{g b}^{\ell}$ & $\mathcal{G}_{w}^{\ell}(0.5)$ & $\mathcal{G}_{w}^{\ell}(2)$ & $\mathcal{G}_{w}^{\ell}(4)$ & $\mathcal{G}^{\ell}(0.5)$ & $\mathcal{G}^{\ell}(2)$ & $\mathcal{G}^{\ell}(4)$ \\
\hline Source 1 & -0.0228 & 0.0123 & -0.0046 & -0.0066 & -0.0105 & -0.0274 & -0.0294 \\
Source 2 & -0.0083 & 0.0045 & -0.0016 & -0.0024 & -0.0038 & -0.0099 & -0.0107 \\
Source 3 & -0.0017 & 0.0010 & -0.0005 & -0.0009 & -0.0007 & -0.0022 & -0.0026 \\
Source 4 & -0.0294 & 0.0150 & -0.0054 & -0.0078 & -0.0144 & -0.0348 & -0.0372 \\
Source 5 & -0.0010 & 0.0005 & -0.0002 & -0.0003 & -0.0005 & -0.0012 & -0.0013 \\
\hline
\end{tabular}

\section{Conclusion}

In this paper, we introduce a first-best Gini multi-decomposition, that is, a multi-decomposition in which the overall Gini ratio is a weighted average of Gini indices per sources and per groups, which is valid whenever subgroup income distributions are non-overlapping. This 
method is well suited to analyze the sources of inequality in a population decomposed in a poor/non-poor manner. In particular, it allows decision makers to easily evaluate taxation schemes, that is, global change in the distribution of source $\ell$, either in a rich group or a poor group. It can also be used to measure the effects on between-group inequalities resulting from a change in income source $\ell$.

In a general manner, the multi-decomposition is a useful property of the extended Gini that helps to deal with the heterogeneity of the agents. Indeed, the fact that a progressive transfer diminishes the overall Gini index is a well-known result in the literature on inequality measurement. Nevertheless, it is not clear how between-group inequalities are affected by a change in a given source of income (or by the subsidization of a given commodity). In this respect, the between-group Gini variations are an important addition to the applied economist toolkit since they measure the impact of tax reforms on between-group inequalities and overall inequality.

\section{Appendix}

In this appendix, we provide guidelines to derive the expressions of Gini variations (16), (19), (20), (21) and (22).

\section{Equation (16):}

This result was established by Lerman and Yitzhaki (1985). They do not provide the demonstration since it is quite straightforward. Indeed, remark that $R_{k k}^{\ell}(\nu)$ and $G_{k k}^{\ell}(\nu)$ are invariant after multiplying each source $\ell$ of group $\Pi_{k}$ by $e_{k k}^{\ell}$. Then, taking the derivative of $S_{k k}^{\ell}, \forall \ell \in\{1,2, \ldots, L\}$, with respect to $e_{k k}^{\ell}$, letting $\frac{\mu_{k}}{\mu_{k}+\mu_{k}^{\ell}+\mu_{k}^{\ell} e_{k k}^{\ell}} \rightarrow 1$ and remembering that $e_{k k}^{\ell} \rightarrow 1^{+}$gives the desired result.

\section{Equation (19):}

Consider a transfer of $e_{k j}^{\ell} \rightarrow 1^{+}$. Then, $\forall \ell \in\{1,2, \ldots, L\}$, we have:

$$
\frac{\left|\mu_{k}^{\ell}-\mu_{j}^{\ell}\right|}{\mu_{k}^{\ell}+\mu_{j}^{\ell}} \cdot \frac{e_{k j}^{\ell}\left(\mu_{k}^{\ell}-\mu_{j}^{\ell}\right)}{\left(\mu_{j}-\mu_{j}^{\ell}\right)+e_{k j}^{\ell} \mu_{j}^{\ell}+\left(\mu_{k}-\mu_{k}^{\ell}\right)+e_{k j}^{\ell} \mu_{k}^{\ell}} \rightarrow G_{k j}^{\ell} S_{k j}^{\ell}
$$

and $\forall \ell \neq h \in\{1,2, \ldots, L\}$ :

$$
\frac{\left|\mu_{k}^{h}-\mu_{j}^{h}\right|}{\mu_{k}^{h}+\mu_{j}^{h}} \cdot \frac{\mu_{k}^{h}+\mu_{j}^{h}}{\left(\mu_{j}-\mu_{j}^{\ell}\right)+\left(\mu_{k}-\mu_{k}^{\ell}\right)+e_{k j}^{\ell}\left(\mu_{j}^{\ell}+\mu_{k}^{\ell}\right)} \rightarrow G_{k j}^{h} S_{k j}^{h} .
$$

Taking the derivative of (A1) with respect to $e_{k j}^{\ell}$, and that of (A2) $\forall h \neq \ell \in\{1,2, \ldots, L\}$ with respect to $e_{k j}^{\ell}$, and then letting $e_{k j}^{\ell} \rightarrow 1^{+}$yields

$$
\frac{\mu_{j}+\mu_{k}}{\left(\mu_{j}-\mu_{j}^{\ell}\right)+e_{k j}^{\ell} \mu_{j}^{\ell}+\left(\mu_{k}-\mu_{k}^{\ell}\right)+e_{k j}^{\ell} \mu_{k}^{\ell}} \rightarrow 1,
$$


which helps to complete (19).

\section{Equation (20):}

Consider a transfer of $e_{k k}^{\ell}$, which only concerns group $\Pi_{k}$. Then, $\forall h \neq \ell \in\{1,2, \ldots, L\}$, we have:

$$
\begin{aligned}
& \frac{\left|\mu_{k}^{\ell} e_{k k}^{\ell}-\mu_{j}^{\ell}\right|}{\mu_{k}^{\ell} e_{k k}^{\ell}+\mu_{j}^{\ell}} \cdot \frac{\mu_{k}^{\ell} e_{k k}^{\ell}+\mu_{j}^{\ell}}{\mu_{j}+\left(\mu_{k}-\mu_{k}^{\ell}\right)+\mu_{k}^{\ell} e_{k k}^{\ell}} \rightarrow G_{k j}^{\ell} S_{k j}^{\ell} \\
& \frac{\left|\mu_{k}^{h}-\mu_{j}^{h}\right|}{\mu_{k}^{h}+\mu_{j}^{h}} \cdot \frac{\mu_{k}^{h}+\mu_{j}^{h}}{\mu_{j}+\left(\mu_{k}-\mu_{k}^{\ell}\right)+\mu_{k}^{\ell} e_{k k}^{\ell}} \rightarrow G_{k j}^{h} S_{k j}^{h} .
\end{aligned}
$$

The income receivers are the individuals of the poorest group, that is, $\Pi_{k}$. Then, we may assume that: $\left|\mu_{k}^{\ell} e_{k k}^{\ell}-\mu_{j}^{\ell}\right|=-\mu_{k}^{\ell} e_{k k}^{\ell}+\mu_{j}^{\ell}$. Remembering that $e_{k k}^{\ell} \rightarrow 1^{+}$, this yields:

$$
\begin{gathered}
\frac{\mu_{j}+\mu_{k}}{\mu_{j}+\left(\mu_{k}-\mu_{k}^{\ell}\right)+e_{k k}^{\ell} \mu_{k}^{\ell}} \rightarrow 1, \\
\frac{\mu_{j}^{\ell}-\mu_{k}^{\ell} e_{k k}^{\ell}}{\mu_{j}^{\ell}+\mu_{k}^{\ell}} \rightarrow G_{k j}^{\ell},
\end{gathered}
$$

which helps to find (20).

\section{Equation (22):}

After the transfer, with $e_{k k}^{\ell} \rightarrow 1^{+}$, we have:

$$
\frac{\mu_{k}-\mu_{k}^{\ell}+\mu_{k}^{\ell} e_{k k}^{\ell}}{\sum_{j \neq k} \frac{n_{j}}{n} \mu_{j}+\frac{n_{k}}{n}\left(\mu_{k}-\mu_{k}^{\ell}+\mu_{k}^{\ell} e_{k k}^{\ell}\right)} \rightarrow \frac{\mu_{k}}{\mu} .
$$

In order to derive (22), we must compute the following partial derivatives:

$$
\begin{gathered}
\frac{\partial C_{k k}^{\ell}(\nu)}{\partial e_{k k}^{\ell}}=P_{k}^{2}\left(\frac{\mu_{k}^{\ell}}{\mu}-\frac{\mu_{k}}{\mu} P_{k} \frac{\mu_{k}^{\ell}}{\mu}\right) R_{k k}^{\ell}(\nu) G_{k k}^{\ell}(\nu) S_{k k}^{\ell} \\
+P_{k}^{2}\left(\frac{\mu_{k}}{\mu}\right)\left(R_{k k}^{\ell}(\nu) G_{k k}^{\ell}(\nu) S_{k k}^{\ell}-R_{k k}^{\ell}(\nu) G_{k k}^{\ell}(\nu) S_{k k}^{\ell} S_{k k}^{\ell}\right) \\
\frac{\partial C_{k k}^{h}(\nu)}{\partial e_{k k}^{\ell}}=P_{k}^{2}\left(\frac{\mu_{k}^{\ell}}{\mu}-\frac{\mu_{k}}{\mu} P_{k} \frac{\mu_{k}^{\ell}}{\mu}\right) R_{k k}^{\ell}(\nu) G_{k k}^{\ell}(\nu) S_{k k}^{\ell} \\
+P_{k}^{2}\left(\frac{\mu_{k}}{\mu}\right)\left(-R_{k k}^{h}(\nu) G_{k k}^{h}(\nu) S_{k k}^{h} S_{k k}^{\ell}\right) \\
\frac{\partial C_{j j}^{h}(\nu)}{\partial e_{k k}^{\ell}}=-P_{j}^{2} \frac{\mu_{j}}{\mu} P_{k} \frac{\mu_{k}^{\ell}}{\mu} R_{j j}^{h}(\nu) G_{j j}^{h}(\nu) S_{j j}^{h}
\end{gathered}
$$




$$
\frac{\partial C_{j j}^{h}(\nu)}{\partial e_{k k}^{\ell}}=-P_{j}^{2} \frac{\mu_{j}}{\mu} P_{k} \frac{\mu_{k}^{\ell}}{\mu} R_{j j}^{\ell}(\nu) G_{j j}^{\ell}(\nu) S_{j j}^{\ell}
$$

\section{Equation (23):}

The poorest group is $\Pi_{k}$, we then assume that: $\left|\mu_{k}^{\ell} e_{k k}^{\ell}-\mu_{j}^{\ell}\right|=-\mu_{k}^{\ell} e_{k k}^{\ell}+\mu_{j}^{\ell}$. Remembering that $e_{k k}^{\ell} \rightarrow 1^{+}$, this yields:

$$
C_{j k}^{\ell}=2 P_{j} P_{k} \frac{-\mu_{k}^{\ell} e_{k k}^{\ell}+\mu_{j}^{\ell}}{2 \sum_{j \neq k} \frac{n_{j}}{n} \mu_{j}+\frac{n_{k}}{n}\left(\mu_{k}-\mu_{k}^{\ell}+\mu_{k}^{\ell} e_{k k}^{\ell}\right)} \rightarrow \frac{-\mu_{k}^{\ell}+\mu_{j}^{\ell}}{2 \mu} .
$$

In order to obtain (23), we take the following partial derivatives:

$$
\begin{gathered}
\frac{\partial C_{j k}^{\ell}}{\partial e_{k k}^{\ell}}=-2 P_{j} P_{k} \frac{\mu_{k}^{\ell}}{2 \mu}-2 P_{j} P_{k} 2 P_{k} G_{j k}^{\ell} \frac{\mu_{j}^{\ell}+\mu_{k}^{\ell}}{2 \mu} \cdot \frac{\mu_{k}^{\ell}}{2 \mu} \\
\frac{\partial C_{j k}^{h}}{\partial e_{k k}^{\ell}}=-2 P_{j} P_{k} 2 P_{k} \frac{\mu_{k}^{\ell}}{2 \mu} G_{j k}^{h} \frac{\mu_{j}^{h}+\mu_{k}^{h}}{2 \mu} .
\end{gathered}
$$

\section{References}

[1] Barrett, G. and P. Pendakur (1995), Asymptotic Distributions for the Generalised Gini Family of Inequality and Welfare Indices, Canadian Journal of Economics, 28(4b), 1043-1055.

[2] Bhattacharya, N. and B. Mahalanobis (1967), Regional disparities in household consumption in India, Journal of the American Statistical Association, 62, 143-161.

[3] Bossert, W. and C. D'Ambrosio (2006), Reference Groups and Individual Deprivation, Economics Letters, 90(3), 421-426.

[4] Bourguignon, F. (1979), Decomposable Inequality Measures, Econometrica, 47, 901920.

[5] Chameni, C. (2007), The multi-decomposition of the Hirschman-Herfindahl index: measuring household inequality in Cameroon, 1996-2001, Applied Economics letters, $14,27-37$.

[6] Cowell, F. (1989), Sampling Variance and Decomposable Inequality Measures, Journal of Econometrics, vol. 42, 27-41.

[7] Dagum, C. (1987), Measuring the Economic Affluence Between Populations of Income Receivers, Journal of Business and Economic Statistics, 5(1), 5-12.

[8] Dagum, C. (1997), Decomposition and Interpretation of Gini and the Generalized Entropy Inequality Measures, Proceedings of the American Statistical Association, Business and Economic Statistics Section, 157th Meeting, 200-205. 
[9] Davidson, R. and E. Flachaire, (2007), Asymptotic and Bootstrap Inference for Inequality and Poverty Measures, Journal of Econometrics, 141, 141-166.

[10] Davidson, R. (2007), Reliable Inference for the Gini Index, Working paper, McGill University.

[11] Deutsch, J. and J. Silber (1999), Inequality Decomposition by Population Subgroups and the Analysis of Interdistributional Inequality, in Silber, J. (ed.), Handbook of Income Inequality Measurement, Kluwer Academic Publishers, 163-186.

[12] Duclos, J.-Y., P. Makdissi and Q. Wodon (2005), Poverty-Dominant Transfer Programs: The Role of Targeting and Allocation Rules, Journal of Development Economics, $77(1), 53-73$.

[13] Fei, J. C. H., Ranis, G. and S. W. Y Kuo (1978), Growth with Equity - The Taiwan Case, Oxford, Oxford University Press.

[14] Fields G. (1979), Income Inequality in Urban Columbia : A decomposition Analysis, Review of Income and Wealth, 25(3), 327-341.

[15] Gini, C. (1916), Il concetto di transvariazione e le sue prime applicazioni, Giornale degli Economisti e Rivista di Statistica, in : C. Gini (Eds.) 1959, 21-44.

[16] Lambert, P. J. and R. J. Aronson (1993), Inequality Decomposition Analysis and the Gini Coefficient Revisited, The Economic Journal, 103, 1221-1227.

[17] Lerman, R. and S. Yitzhaki (1985), Income Inequalities Effects by Income Source: A New Approach and Applications to United States, Review of Economics and Statistics, $67,151-156$.

[18] Lerman, R. and S. Yitzhaki (1991), Income Stratification and Income Inequality, Review of Income and Wealth, 37(3), 313-329.

[19] Makdissi, P. and Q. Wodon (2007), Poverty-Reducing and Welfare-Improving Marginal Public Price and Price Cap Reforms, Journal of Public Economic Theory, 9, 683-698.

[20] Mookherjee, D., and A. Shorrocks (1982), A Decomposition Analysis of the Trend in UK Income Inequality, The Economic Journal, 92, 886-902.

[21] Mussard, S. (2004), Décompositions Multidimensionnelles du Rapport Moyen de Gini, Ph.D., University of Montpellier I.

[22] Mussard, S. (2006), Une réconciliation entre la décomposition en sous-groupe et la décomposition en sources de revenu de l'indice de Gini. La multi-décomposition de l'indicateur de Gini, Annales d'Economie et de Statistique, 81, 1-25. 
[23] Pyatt, G. (1976), On the Interpretation and Disaggregation of Gini Coefficients, Economic Journal, 86, 243-25.

[24] Rao, V.M. (1969), Two Decompositions of Concentration Ratio, Journal of the Royal Statistical Society, Series A 132, 418-425.

[25] Sastry, D.V.S. and U.R. Kelkar (1994), Note on the Decomposition of Gini Inequality, Review of Economics and Statistics, LXXVI, 584-585.

[26] Shorrocks A. F. (1980), The Class of Additively Decomposable Inequality Measures, Econometrica, 48, 613-625.

[27] Shorrocks A. F. (1982), Inequality Decomposition by Factor Component, Econometrica, 50, 193-211.

[28] Shorrocks, A.F. (1999), Decomposition Procedures for Distributional Analysis: A Unified Framework Based on the Shapley Value, Mimeo, University of Essex.

[29] Silber, J. (1989), Factor Components, Population Subgroups and the Computation of the Gini Index of Inequality, Review of Economics and Statistics, 71, 107-115.

[30] Silber, J. (1993), Inequality Decomposition by Income Source : a Note, Review of Economics and Statistics, 75(3), 545-547.

[31] Silber, J. (1998), On Inequality in Consumption Expenditures. The case of Switzerland, Swiss Journal of Economics and Statistics, 124(41), 545-564.

[32] Yao, S. (1999), On the decomposition of Gini coefficients by population class and income source: a spreadsheet approach and application, Applied Economics, 31, 12491264 .

[33] Yitzhaki, S. (1979), Relative Deprivation and the Gini Coefficient, Quarterly Journal of Economics, 93, 321-324.

[34] Yitzhaki, S. (1983), On an Extension of the Gini Inequality Index, International Economic Review, .

[35] Yitzhaki, S. (2002), Do we Need a Separate Poverty Measurement?, European Journal of Political Economy, 18, 61-85. 